\title{
Manual de experimentación como apoyo didáctico en la enseñanza de las ciencias naturales a nivel de Educación Inicial
}

\author{
Experimentation manual as didactic support in the teaching of natural \\ sciences at the Initial Education level
}

$\begin{array}{lr}\text { Pablo Ramón Lorca Noriega } & \text { Artículo recibido en octubre } 2019 \\ \text { pablorcanoriega@gmail.com } & \text { Arbitrado en noviembre } 2019 \\ \text { Código ORCID: 0000-0002-1981-1095 } & \text { Publicado en enero 2020 } \\ \text { Ministerio del Poder Popular para la Educación, Venezuela } & \end{array}$

\section{Resumen}

La presente investigación consistió en diseñar una propuesta basada en la experimentación como apoyo didáctico en la enseñanza de las ciencias naturales en la educación inicial dirigido a los docentes de los centros de educación inicial del municipio Naguanagua del estado Carabobo. Estuvo fundamentada teóricamente bajo Piaget y la teoría socio-pedagógica de Díaz y Hernández. La metodología empleada se enmarcó en la modalidad de proyecto factible, sustentado en un análisis documental y estudio de campo. La población estuvo constituida por 30 docentes, de los cuales se tomó una muestra por azar simple de 15 docentes, se utilizó una encuesta bajo la modalidad de escala de Likert. Los principales resultados muestran deficiencias en la enseñanza de las ciencias naturales mediante la experimentación en niños y niñas, además de actividades realizadas por ellos de manera espontánea, situación que conlleva a trabajar actividades donde se introducen símbolos sin referencias a sus significados.

\footnotetext{
Abstract

The present investigation consisted of designing a proposal based on experimentation as a didactic support in the teaching of natural sciences in initial education, aimed at teachers of initial education centers in the Naguanagua municipality of Carabobo state. It was theoretically based under Piaget and the socio-pedagogical theory of Díaz and Hernández. The methodology used was framed in the feasible project modality, supported by a documentary analysis and field study. The population consisted of 30 teachers, from which a simple random sample was taken from 15 teachers, a survey using the Likert scale modality was used. The main results show deficiencies in the teaching of natural sciences through experimentation in boys and girls, in addition to activities carried out by them spontaneously, a situation that leads to working activities where symbols are introduced without references to their meanings.
}

Palabras clave:

Ciencias naturales; enseñanza; experimentación
Keywords:

Natural sciences; teaching; experimentation 


\section{INTRODUCCIÓN}

A lo largo de los últimos años las investigaciones se han enmarcado en conseguir la forma más adecuada de aprender acerca de los fenómenos naturales presentes en el ambiente. El currículo de educación inicial, con respecto a la enseñanza de tipo no formal, ha llegado a las conclusiones que los niños y niñas mucho antes de ingresar a un sistema de estudio formal o no formal construyen conocimiento informal respecto a los temas y su observación en la vida cotidiana.

En tal sentido, dicho conocimiento debe ser implementado en las estrategias claras que utilicen el ambiente físico, sus cambios visibles, llevándolos a las aulas de clases, con el fin de que se empleen procesos de construcción físicos en donde se comprendan algunos fenómenos que rodean al niño o la niña en su entorno ambiental.

Por consiguiente, comenzar por tareas sencillas donde el aprendizaje se desarrolle en un ambiente que propicie la participación activa y crítica de los niños. Esto implica cambiar las concepciones actuales, las cuales se ubican entre dos enfoques: el convencional o tradicional que va de la copia a la resolución en físico de las diferentes actividades donde cada clase se organiza en torno a una secuencia de lecciones que deben aprenderse a través de la ejercitación mecánica, memorización y sistemática de un modelo; el activista en el cual no se explotan todas las potencialidades de los niños y niñas a favor de un respeto mal entendido a su proceso de aprendizaje, que olvida el carácter intencional de la enseñanza y necesaria orientación que el docente debe ejercer.

Al respecto, Araque y Monsalve (2014) señalan:

Enseñar es una tarea que dignifica la persona, el logro de una enseñanza capaz de proporcionar a los estudiantes la posibilidad de aprender a aprender adquiere una importancia de primer orden, donde el alumno no aprende solo, sino con la actividad auto estructurante del sujeto que estará mediada por la influencia de los otros, y el aprendizaje se convierte en una actividad de reconstrucción de los saberes de una cultura. (p. 22)

Lo antes expuesto hace alusión a que el rol del docente es el de actuar como mediador 0 intermediario entre los contenidos conceptuales, actitudinales y procedimentales que forman parte de la actividad constructiva que desarrollan los alumnos para asimilarlos. Por otra parte no se puede dejar de reconocer que la enseñanza debe individualizarse en el sentido de permitir a cada estudiante trabajar con independencia y a su propio ritmo, promoviendo la colaboración y el trabajo grupal.

El currículo de educación inicial (2005) señala: en el sistema educativo venezolano el niño y la niña parte en una primera etapa de su vida en nivel inicial, este es considerado uno de los niveles más influyentes, en donde se desarrolla la cadena del aprendizaje, este debe estar afianzado en sus bases, implementando todos los ejes para lograr generaciones futuras que adopten patrones científicos, psicológicos y lo utilicen en pro de nuevas sociedades. Entendiendo por etapa escolar al periodo acorde a su edad el cual está estructurado de la siguiente manera: 0 a 6 años educación inicial.

Siguiendo el orden de ideas, el currículo de educación inicial. (2005)

Plantea que el niño y la niña son el centro y autores de su propio aprendizaje con atención a las peculiaridades individuales y el 
respeto como seres únicos dentro de contexto familiar y comunitario.

Es donde se debe tomar la función y rol del docente del aula, recaudando todas las inquietudes brindadas por los niños y niñas basándose en su autonomía. (p.14)

En otras palabras, el niño y la niña toman las experiencias previas adquiridas como punto de partida para crear herramientas que llenen esas necesidades dando relevancia a procesos afectivos entre él y su entorno.

Los ambientes educativos son aquellos que dictan, que quieren hacer, como lo van hacer, que quieren aprender partiendo desde sus experiencias previas, eventos cotidianos donde el niño y la niña sea capaz de representar o reproducir los fenómenos observados, con la finalidad de dar respuesta con la práctica escolar lo que observa en los distintos ambientes en donde se desarrolla su ser.

El currículo de Educación Inicial (2005), se plantea "el elemento esencial del proceso de socialización es el propio niño y la propia niña, su capacidad para relacionarse con el entorno $e$ ir accediendo gradualmente a niveles de mayor conocimiento y de nexos sociales cada vez más complejos, a través de un interacción dinámica" (s.n). Es allí en donde su propia actividad, la de sus pares o iguales y la de los adultos responsables de su desarrollo facilitaran la construcción de nuevos patrones sociales.

En tal sentido, el docente en educación inicial debe contar con estrategias que promuevan el desarrollo del conocimiento de un nivel a otro mediante experiencias de aprendizaje activo, lo que pedagógicamente implica brindar al niño y a la niña la oportunidad de observar, manipular, experimentar, que se planteen interrogantes y traten de buscar sus propias respuestas.

Entendiendo por experimentar en el nivel de educación inicial la relación existente en ambientes convencionales y no convencionales con la finalidad de generar un aprendizaje activo, este se convierte en un aprendizaje principal por el cual los niños y niñas desarrollan, construyen y forman el conocimiento, creando relaciones existentes entre el ambiente educativo y su ambiente cotidiano.

Esto ocurre mediante un proceso interacción social, en donde una adecuada creación de un ambiente de apoyo interpersonal y social relevante para que estos tengan total libertad de manipular materiales, hacer elecciones, tomar decisiones, compartir en grupos, conversar o reflexionar acerca lo que hacen y observan en el entorno que lo rodea.

El propósito de enseñar ciencias es desarrollar la capacidad del niño para entender la naturaleza de su entorno. Los niños y los adultos debemos comprender que lo que se necesita para ser científico lo tenemos potencialmente cada uno de nosotros. La intención principal es formar seres humanos con una visión integral, promoviendo el desarrollo de habilidades de pensamiento científicas en los niños; como el cuestionar y reflexionar sobre los fenómenos naturales que suceden a nuestro alrededor, mediante la investigación de las causas que los generan; hecho que le permitirá desarrollar una actitud científica y convertirse en un ser reflexivo, crítico y analítico; capaz de seguir un método para realizar investigaciones a partir de la información disponible, formular hipótesis y verificar las mismas mediante la experiencia. 
Según el currículo de educación inicial (2005):

\begin{abstract}
Estas interacciones se conocen o presentan como la forma de experimentar, en donde se favorece al aprendizaje significativo con el cual el niño y la niña encuentren relación con los conocimientos previos, con su contexto social y cultural con el fin de promover el aprendizaje participativo en ese grupo etario a manera de que los niños y niñas se relaciones y actúen también como mediadores de los procesos de sus compañeros(as). (p. 25)
\end{abstract}

En este orden de ideas la función de él o la docente a cargo del desarrollo cognitivo y el aprendizaje de niño y niñas entre los 6 años está enmarcado primordialmente en una planificación, en donde él como adulto activo media, facilita, propicia, coordina y planifica el proceso de aprendizaje, evitando buscar un aprendizaje de producto u homogéneo sino crear o planificar experiencias de aprendizaje base que le oriente a una evaluación formativa del proceso.

Es por ese motivo que en la actualidad es un deber emplear estrategias novedosas para la integración social y cultural, mediante juego o programas de aprendizajes novedosos que utilicen los métodos de experimentación o manipulación de elementos que generen un aprendizaje significativo.

Bello (2012) señala que la integración de los nuevos conocimientos a los ya existentes es un proceso muy complejo que requiere de múltiples y variadas situaciones de aprendizaje, tiempo y oportunidades para que los niños y niñas pongan en juego ciertas acciones: comparar, establecer relaciones, transformar, analizar, anticipar los resultados, el proceso a seguir, ensayar una posible solución, razonar y justificar los resultados.

La integración de conocimientos permite crear relación entre lo que cotidianamente vive el niño y la niña en su ambiente social y como el docente en su rol de orientador o mediador puede dar respuestas necesarias para satisfacer las necesidades de los niños y niñas. Sin dejar de lado las herramientas tradicionales como el uso adecuado del lenguaje o de recursos ilustrativos.

Por otra parte, en el marco de entender en esta sección introductoria algunos antecedentes que soportan la problemática investigada, se encuentra el aporte de Romero (2015), y su propuesta "Estrategias didácticas con material de provecho para el desarrollo motriz fino del niño y la niña C.E.I Bárbula II del grupo etario 3". En líneas generales, tuvo como objetivo general interpretar las estrategias didácticas con material de provecho para desarrollo motriz fino del niño y la niña, formando valores ecológicos para la conservación de sus espacios ambientales.

La presente investigación guardó relación con el estudio en curso, ya que se aspira que los docentes o maestros del nivel inicial logren que los niños y niñas muestren el desarrollo necesario del uso de las ciencias naturales, mediante pequeñas vivencias pedagógicas que cumplan con el nivel de formación de los estudiantes.

Martínez y Álvarez (2015) en su investigación titulada "La importancia de los experimentos pautados en la educación básica" exponen la experimentación en el aula como un instrumento pautado de enseñanza-aprendizaje, que el maestro debe utilizar para interrelacionar la teoría y la práctica. Al mismo tiempo, el alumnado evoluciona a partir de sus inteligencias 
múltiples y de sus aprendizajes previos e intuitivos para llegar a construir su propio conocimiento a largo plazo.

Por su parte, Pérez (2013) en su investigación titulada "Uso de las ilustraciones como estrategias en la enseñanza - aprendizaje de la física: cinemática" En esta se realza la importancia de usar referentes cotidianos en la didáctica de las ciencias, en particular, en la Enseñanza de la Física. Esta ha sido una preocupación constante desde la década de los sesenta, pues diversos autores y organizaciones internacionales han propuesto metodologías y estrategias para incrementar el logro y la motivación de los estudiantes de nivel básico, destacando siempre el uso de los recursos experimentales y de la experimentación en la Enseñanza de las Ciencias Naturales.

En esta investigación se dio realce a que siempre se debe tener una amplia relación entre las teorías visibles y las prácticas de laboratorios o experimentales bien sea el caso en efecto, si se trata de una ley que no ha sido estudiada en clase, mediante la ilustración de una demostración de aula o con una guía de laboratorio bien orientada el estudiante puede obtener una serie de datos experimentales que, luego de tabulados y llevados a un gráfico, le permitirán llegar con más o menos esfuerzo a la ley buscada.

Dorante (2015) en su trabajo titulado "Diseño de una guía sobre estrategias didácticas para fortalecer la enseñanza y aprendizaje de la física", señala que el proceso de enseñanza y aprendizaje de la física en educación media general y particularmente en tercer año de este nivel educativo, constituye un proceso complejo que amerita de una praxis pedagógica centrada en los procesos y no en los resultados. En tal sentido, la guía de estrategias de razonamiento inductivo presentada a los docentes del municipio Sucre estado Portuguesa, buscó principalmente contribuir en el desarrollo de situaciones de aprendizajes amenos, significativos e innovadoras, que propicien el interés por esta ciencia universal.

Si bien es cierto que la guía de estrategias de razonamiento inductivo presentada en esta investigación, no solventa definitivamente la situación problema de la cual se partió en este estudio, no menos cierto es que constituye un aporte para minimizar las dificultades que se les presenta a los docentes en la enseñanza de la física, pero a su vez representa una valiosa herramienta de carácter didáctico para promover en las y los estudiantes aprendizajes verdaderamente significativos.

De esta manera, los antecedentes de la investigación contextualizan y justifican la importancia de implementar o facilitar guía o manuales que busquen dar realce a los estudios de las ciencias naturales implementando los 4 ejes primordiales para el proceso de formación, brindando a docentes y maestras del nivel herramientas necesarias que utilicen como norte la motivación experimental siendo compartida en los 4 ambientes físicos dentro del aula de clases.

Armar y construir, representar e imitar, expresar y crear, representar e imitar son los ambientes físicos en donde los niños y niñas deben contar con la herramientas necesarias para construir el aprendizaje, formado mediante el juego de intercambio de roles o de forma experimental aplicando el conocimiento cotidiano para generar un efecto o cambio en su desarrollo cognitivo.

Como menciona Bello (2012), si la relación cotidiano - efecto se desarrolla a nivel de ciencias naturales, entonces se afianzaría las bases de las cadena. Partiendo desde el hacer para comprender, la motivación e inquietud del niño y de la niña para sacar provecho a las generaciones futuras. 
En los centros de educación inicial del municipio Naguanagua no se escapa de esta problemática, puesto que aunque la planificación y programación de las actividades diarias para el niño y la niña estén acordes a su edad, no son eficaces a largo tiempo o no son estrategias significantes para el proceso de construcción del conocimiento del niño y de la niñas en relación a la interacción del ambiente o el espacio que lo rodea.

Allí es necesario hacer uso de la experimentación como herramienta de enseñanza para el estudio de los fenómenos como la formación de rayos, cambios físicos, cambios climáticos, rotación y traslación, entre otros, no solo de forma ilustrada u oral sino también de forma que el niño y niña cree patrones de relación mediante el hacer y el deber. Por tal motivo que se permite construir o buscar alternativas en pro del aprendizaje del niño generando habilidades de pensamiento que le permitan enfrentar el proceso aprendizaje y además desarrollar, ejecutar, perfeccionar y despertar las capacidades mentales del mismo.

Es así como surgió la necesidad de analizar la estrategia de una guía de experimentación como apoyo didáctico para la enseñanza de las ciencias naturales en los centros de educación inicial del municipio Naguanagua, estado Carabobo- Venezuela, y, de esta manera, promover en los docentes y niños (as) una formación inicial con visión estratégica.

Por tal motivo, se buscó diseñar un Manual de experimentación como apoyo didáctico en la enseñanza de las ciencias naturales en la educación inicial. Caso: centros de educación inicial del municipio Naguanagua, estado Carabobo, Venezuela.

Los objetivos específicos del estudio fueron: 1. Diagnosticar las estrategias utilizadas por el docente con respecto a las ciencias naturales en los centros de educación inicial, del municipio Naguanagua del estado Carabobo; 2. Estudiar la factibilidad de la propuesta didáctica basada en la experimentación apoyo didáctico en la enseñanza de las ciencias naturales en la educación inicial dirigida a los docentes de los centros de educación inicial, del municipio Naguanagua del estado Carabobo; 3. Elaborar una material didáctico basado en la experimentación para el apoyo didáctico en la enseñanza de las ciencias naturales en la educación inicial dirigida a los docentes de los centros de educación inicial, del municipio Naguanagua del estado Carabobo, Venezuela.

\section{MÉTODO}

Esta investigación se enmarcó en la modalidad de proyecto factible, sustentando un estudio descriptivo y de campo. Según Arias (2008)

La investigación de campo es aquella que consiste en la recolección de datos directamente de los sujetos investigados, o de la realidad donde ocurren los hechos (datos primarios), sin manipular o controlar variable alguna, es decir, el investigador obtiene la información pero no altera las condiciones existentes. De allí su carácter de investigación no experimental. (p. 31)

En este sentido, para realizar esta investigación, se fundamentó en un estudio de campo en donde se describió el contexto o situación, por consiguiente, el diseño de investigación fue descriptivo. Para Sampieri (2003) los estudios descriptivos buscan especificar las propiedades, características y los perfiles importantes de persona, grupos, comunidades o cualquier otro fenómeno que se someta a un análisis.

Generalmente, el diseño de investigación descriptivo se caracteriza 
porque el investigador está en contacto directo con el objeto de estudio, en otras palabras, la investigación se realiza en el mismo lugar de los acontecimientos, con el fin de responder a la necesidad de implementar un manual de experimentación como herramienta para el aprendizaje de las ciencias naturales en la educación inicial. Así se propicia la generación de cambios en los procesos de facilitación del aprendizaje y se suministran al docente las estrategias y medios de formalidad para que los niños y niñas del nivel inicial afiancen el conocimiento científico.

Por otro lado, la población de esta investigación estuvo constituida por 30 docentes de los centros de educación inicial del municipio Naguanagua del estado Carabobo, Venezuela.

También conviene destacar que una muestra representativa es aquella que por su tamaño y características similares a las del conjunto y permite hacer inferencias o generalizar los resultados al resto de la población con un margen de error conocido.

Para el presente estudio la muestra estuvo constituida por el $30 \%$ de los profesionales en educación inicial. Lo anterior lleva a considerar esta muestra como de tipo ofimático o intencional.

\section{RESULTADOS}

La propuesta didáctica para la enseñanza de las ciencias naturales utilizando la experimentación como herramienta y apoyo didáctico para los docentes del tercer nivel de educación inicial, haciendo uso del material completo, el cual busca brindar al docente a cómo desarrollar procesos fundamentales como: la creatividad, la abstracción y el desarrollo del pensamiento científico. Por consiguiente, se define como una herramienta de fácil acceso al docente de educación inicial para abordar nociones básicas de experimentación científica en el tercer nivel del jardín de infancia, al mismo tiempo favorece el desarrollo de habilidades del pensamiento en los niños y niñas de educación inicial para: observar, identificar, experimentar, discutir sobre el aprendizaje en diversas situaciones fuera del jardín de infancia.

Esta propuesta partió de la finalidad de dar a conocer a los docentes del tercer nivel de educación inicial una estrategia concreta basada en la experimentación como apoyo didáctico en la enseñanza de las ciencias naturales. A través de esta se pretende: 1 . Proporcionar a los docentes estrategias prácticas, innovadoras y creativas que ayuden a su vez la capacidad de comprender el proceso cognoscitivo progresivamente en los niños y niños. 2. Desarrollar sistemática y progresivamente el pensamiento crítico, creador, innovador y científico en los niños y niñas.

La propuesta tuvo como principal propósito implementar el conocimiento científico con el fin de minimizar la problemática que se presenta en la enseñanza y aprendizaje de las ciencias naturales en el tercer nivel de educación inicial.

Así mismo, se justifica porque en los centros de educación inicial la mayoría de los docentes explican los contenidos referentes a las ciencias naturales presentes en el currículo de educación inicial, de una manera abstracta, donde solo utilizan procesos de comparación con fenómenos vistos y no se recrea de manera experimental, sino se explica de forma mecánica, siendo identificados en producciones orales y escrita.

En tal sentido, el manual es un apoyo didáctico dirigido a docentes capaces de 
promover en los niños el manejo de sus propias herramientas para aprender la responsabilidad vista desde sus propios sentidos, el sentir razonable a su propia conceptualización, estructuración mental, así con sus necesidades e intereses, convirtiendo al docente en el facilitador, orientador y mediador del aprendizaje.

Por tal motivo, esta propuesta utiliza y partió de la experimentación como herramienta, la cual permitirá a los docentes y niños aprender la relación constante entre el aprendizaje científico y el intercambio entre el ambiente.

Es así como "Experimento de la mano con la ciencia" se convierte en un manual de carácter didáctico donde se pretendió combinar, la experimentación, el uso de material de provecho y la creación de prototipos para facilitar a los docentes como a los niños de una manera fácil, sencilla y divertida de enseñar y aprender ciencias naturales.

En los centros de educación inicial el abordaje de temas científicos están enmarcados en una fundamentación básica en cuanto a estrategias atañen, muchas veces porque no se le facilita las herramientas o manuales necesarios o el desconocimiento con respecto al contenido son algunos de los factores que conllevan a un déficit en el pensamiento científico.

Ahora bien, muchos de estos profesores han recibido cursos de formación para desempeñarse como docentes, sin embargo no han reflexionado sobre lo que significa saber enseñar en el aula. Puesto que no han logrado articular los saberes y el aprendizaje con la cotidianidad en el aula para generar procesos de cambios en la enseñanza y en el aprendizaje. Sobre todo en los contenidos científicos, que forman parte del saber hacer del futuro del niño y la niña, el cual está íntimamente relacionado con la construcción de un aprendizaje significativo y estratégico.

De esto se genera la necesidad de plantear un manual como estrategias a los docentes para que en su rol de mediador de los aprendizajes pueda innovar, organizar el material para el proceso instruccional a fin de lograr un proceso de enseñanza que será fructífero en la medida que permita la construcción de conocimiento integrador, a través de la aplicación de estrategias que no son otra cosa que un agente de enseñanza utilizada de forma creativa, reflexiva y flexible para promover la obtención de un aprendizaje significativo en los estudiantes.

Cabe destacar que la propuesta deja clara la posibilidad al docente en compañía de los niños y niñas de aprovechar cualquier otra estrategia, considerando el ambiente escolar, la capacidad creadora y la planificación del trabajo, la cual es conveniente revisar continuamente a fin de formular juicios oportunos sobre lo que los niños y niñas están logrando. De allí que el objetivo general de dicha propuesta fue: Facilitar a los docentes una estrategia para desarrollar habilidades del pensamiento práctico y creativo al momento de enseñar las ciencias naturales en los niños y niñas del tercer nivel de educación inicial.

Para alcanzar ese objetivo se realizaron los siguientes objetivos específicos, que fueron: 1. Proporcionar actividades prácticas experimentales significativas que fomenten al niño y a la niña el pensamiento científico. 2. Realzar la participación significativa en trabajos de pequeños grupos. 3. Estimular el pensamiento científico en los niños y niñas. 4. Observar por medio de la experimentación un pensamiento científico básico con respecto a las ciencias naturales. 
CONCLUSIONES

En cuanto al nivel de conocimiento de los docentes con respecto al uso de la experimentación para abordar los temas científicos y como apoyo didáctico en sus planificaciones, se detectó que existen debilidades en los docentes al momento de incorporar estas estrategias como herramienta en la planificación con el fin de crear un pensamiento científico en los niños y niñas en el nivel de educación inicial. Tomando en cuenta que la experimentación permite recrear, crear y enriquecer los contenciosos científicos programáticos en el currículo de educación inicial. Generando un aprendizaje significativo al niño y a las niñas, fomentando el debate, la discusión y la observación práctica de los fenómenos orientados al pensamiento lógico científico e innovador.

De igual manera, las estrategias a utilizar por la o los docentes sobre los temas científicos mediante la experimentación, permite la interacción de los cuatros espacios de aprendizaje dentro del salón de clase, así mismo el desempeño del docente en las áreas científicas juega un papel fundamental durante todo el proceso de instrucción de las áreas y contenidos científicos, estableciendo patrones o paradigmas que mejoran la comunicación, habilidades y destrezas de los niños y niñas, las cuales sirven para definir el desarrollo de la práctica educativa en el proceso de enseñanza y aprendizaje.

A lo anterior se le agrega que los docentes manifiestan la necesidad de un manual de experimentación para la producción, utilización e implementación de materiales, prototipos, experimentos, entre otros, para realzar la temática científica en el nivel de enseñanza de manera actualizada en todo momento en el currículo de educación inicial, con el fin de exaltar en los niños y niñas un razonamiento deductivo, lógico y concreto al momento de observar y comparar lo que sucede en su ambiente, llevándolo o recreándolo en el colegio.

Los aspectos señalados plantean el problema de la formación en esta área. En este sentido, se puede afirmar que se requiere de una sólida formación docente para que la práctica pedagógica considere y respete la autonomía del niño e igualmente que las tareas y demandas del aprendizaje estén convenientemente fundamentadas en el contexto cultural y en el nivel evolutivo de éstos.

Así mismo, se requiere de un cambio de actitud para investigar e indagar como los niños observan y comprenden los fenómenos naturales que se presentan en el ambiente con el fin de abordar los contenidos científicos empleando las herramientas científicas necesarias, como la observación, la formulación de hipótesis y comparación en el pensamiento científico. Esto trae como consecuencia el desarrollo de investigaciones sobre las herramientas que los niños y niñas utilizan para dar sentido, comparando y aplicándolas en su vida cotidiana, por ejemplo: cómo comunicarse, de qué manera tomar decisiones y contrastar con mayor facilidad y comprensión el ambiente físico que los rodea.

De igual forma, se le sugiere al docente de educación inicial la reflexión acerca de los nuevos retos y compromisos que se presenten en el desempeño de su profesión. En tal sentido, esta experiencia investigativa le ofrece un apoyo para inventar estrategias como juegos y recursos para la acción de la práctica didáctica. En consecuencia debe reconocerse la importancia de emplear la experimentación y también es necesario el hecho de permitirle al niño conversar sobre sus experiencias previas. 
La interacción práctica, dentro del ambiente de aprendizajes, demuestra que dejar que el niño experimente sobre los fenómenos que observa, permite al docente acceder a su pensamiento significativo. Así, la experimentación es importante porque ofrece la oportunidad de escudriñar los procesos mentales de la mano al proceso didáctico dentro y fuera de las aulas.

\section{REFERENCIAS}

Arias, F. (2008). El Proyecto de Investigación. Introducción a la Metodología Científica. Caracas: Epísteme

Bello, Y. (2012). El lenguaje como herramienta en el aprendizaje de la aritmética en educación inicial caso: tercer nivel del jardín de infancia de la unidad educativa "Moral y Luces". Valencia, Venezuela: Universidad de Carabobo

Currículo de Educación Inicial. (2005). Caracas: Torino

Díaz, F. y Hernández, G. (2001). Estrategias docentes para un aprendizaje significativo. Colombia: McGraw-Hill
Dorante, A. (2015). Diseño de una guía sobre estrategias didácticas para fortalecer la enseñanza y aprendizaje de la física. Valencia, Venezuela: Universidad de Carabobo

Hernández Sampieri, Fernández R y Batista L. (2003). Metodología de la Investigación. (3ra. Ed.). México: McGraw-Hill

Martínez, M. y Álvarez, I. (2015). La importancia de los experimentos pautado en educación básica. Valladolid, España: Universidad de Valladolid, Facultad de Educación de Segovia

Pérez, E. (2013). Uso de las ilustraciones como estrategias en la enseñanza aprendizaje de la física: cinemática. Valencia, Venezuela: Universidad de Carabobo

Piaget, J. (1981). Psicología y educación. Barcelona, España: Ariel

Romero, N. J. (2015).Estrategias didácticas con material de provecho para el desarrollo motriz fino del niño y la niña CEI Bárbula II del grupo etario 3 años (Trabajo de grado). Valencia, Venezuela: Universidad de Carabobo 